\title{
MOSER-TRUDINGER INEQUALITIES OF VECTOR BUNDLE OVER A COMPACT RIEMANNIAN MANIFOLD OF DIMENSION 2
}

\author{
YUXIANG LI, PAN LIU, YUNYAN YANG
}

\begin{abstract}
Let $(M, g)$ be a 2-dimensional compact Riemannian manifold. In this paper, we use the method of blowing up analysis to prove several Moser-Trdinger type inequalities for vector bundle over $(M, g)$. We also derive an upper bound of such inequalities under the assumption that blowing up occur.
\end{abstract}

\section{Introduction and Main results}

Let $(M, g)$ be a 2-dimensional compact Riemannian manifold. One of Fontana's results (see F] says

$$
\sup _{\int_{M}|\nabla u|^{2} d V_{g}=1, \int_{M} u d V_{g}=0} \int_{M} e^{\alpha u^{2}}=\left\{\begin{array}{lll}
<+\infty & \text { if } & \alpha \leq 4 \pi \\
=+\infty & \text { if } & \alpha>4 \pi
\end{array},\right.
$$

which extends Trudinger and Moser's inequalities (see [T, [M]). A weak form of the above inequality is

$$
\log \int_{M} e^{u} d V_{g} \leq \frac{1}{16 \pi} \int_{M}|\nabla u|^{2} d V_{g}+\int_{M} u d V_{g}+C
$$

for all $u \in H^{1,2}(M)$, where $C$ depends only on the geometry of $M$ (see [M], $\underline{\mathrm{A}}$ ). The inequality (1.1) has been extensively used in many mathematical and physical problems, for instance in the problem of prescribing Gaussian curvature ( $\mathrm{Ch}$, [C-Y], D-J-L-W]), the mean field equation and the abelian Chern-Simons model ([D-J-L-W2, [D-J-L-W3, [J-W]), ect.

In this note we want to derive some new Moser-Trudinger type inequalities. We will consider a smooth vector bundle $E$ with metric $h$ and connection $\nabla$ over $M$. Throughout this paper, we do not assume $\nabla h=0$. To simplify the notations, we write

$$
H_{0}=\left\{\sigma \in H^{1,2}(M, E): \nabla \sigma=0\right\},
$$

and $H_{1}=H_{0}^{\perp}$, i.e.

$$
H_{1}=\left\{\sigma \in H^{1,2}(M, E): \int_{M}\langle\sigma, \zeta\rangle d V_{g}=0, \text { for all } \zeta \in H_{0}\right\} .
$$

We state our main results as follows:

Theorem 1.1 Let $(M, g)$ be a 2-dimensional compact Riemannian manifold, $(E, h)$ be a smooth vector bundle over $(M, g), \nabla$ and $H_{1}$ be defined as above. Denote

$$
\mathcal{H}_{1}=\left\{\sigma \in H_{1}: \int_{M}\langle\nabla \sigma, \nabla \sigma\rangle d V_{g}=1\right\} .
$$


Then we have

$$
\sup _{\sigma \in \mathcal{H}_{1}} \int_{M} e^{4 \pi|\sigma|^{2}} d V_{g}<\infty
$$

where $|\sigma|^{2}=\langle\sigma, \sigma\rangle_{h}$, and the constant $4 \pi$ is sharp, which means that for any $\alpha>4 \pi$,

$$
\sup _{\sigma \in \mathcal{H}_{1}} \int_{M} e^{\alpha|\sigma|^{2}} d V_{g}=\infty
$$

As a corollary of Theorem 1.1, we have

Corollary 1.2 There exists a constant $C$ such that

$$
\int_{M} e^{|\sigma|} d V_{g} \leq C e^{\frac{1}{16 \pi} \int_{M}\langle\nabla \sigma, \nabla \sigma\rangle d V_{g}}
$$

holds for all $\sigma \in H_{1}$.

We remark two special cases of Theorem 1.1: If $E$ is a trivial bundle, $e_{i}$ is global basis of $E$, with

$$
\left\langle e_{i}, e_{j}\right\rangle_{h}=h_{i j} \text { and } \nabla e_{i}=0
$$

then we have the following:

Corollary 1.3 Let $(M, g)$ be a 2-dimensional compact Riemannian manifold. Given an positive symmetric matrix $H$, we denote

$$
\mathcal{H}_{1}=\left\{U \in H^{1,2}\left(M, \mathbb{R}^{n}\right): \int_{M} \nabla U H \nabla U^{T} d V_{g}=1, \int_{M} U d V_{g}=0\right\},
$$

then we have

$$
\sup _{U \in \mathcal{H}_{1}} \int_{M} e^{4 \pi U H U^{T}} d V_{g}<+\infty
$$

where $4 \pi$ is sharp.

If $E$ is a trivial line bundle with $\langle e, e\rangle_{h}=f(x)$, Corollary 1.3 is exactly Yang's result $[\mathbf{Y}$.

A complete analogue of Theorem 1.1 is the following:

Theorem 1.4. Let $(M, g)$ be a 2-dimensional compact Riemannian manifold, $(E, h)$ be a smooth vector bundle over $(M, g), \nabla$ and $H_{1}$ be defined as before. Denote

$$
\left.\mathcal{H}_{2}=\left\{\sigma \in H_{1}: \int_{M}\langle\nabla \sigma, \nabla \sigma\rangle+|\sigma|^{2}\right) d V_{g}=1\right\} .
$$

Then we have

$$
\sup _{\sigma \in \mathcal{H}_{2}} \int_{M} e^{4 \pi|\sigma|^{2}} d V_{g}<\infty
$$

where the constant $4 \pi$ is sharp.

The proof of Theorem 1.1 is outlined as follows (the proof of Theorem 1.4 is completely analogous to that of Theorem 1.1, so we omit it). Let us define $J_{\alpha}(\sigma)=\int_{M} e^{\alpha|\sigma|^{2}} d V_{g}$. We first 
show that the sup of $J_{\alpha}$ can be attained in $\mathcal{H}_{1}$ if $\alpha<4 \pi$. So we can choose $\alpha_{k}$ converging to $4 \pi$ increasingly, and $\sigma_{k} \in \mathcal{H}_{1}$ satisfying

$$
J_{\alpha_{k}}\left(\sigma_{k}\right)=\sup _{\sigma \in \mathcal{H}_{1}} J_{\alpha_{k}}(\sigma)
$$

Denote $c_{k}=|\sigma|\left(x_{k}\right)=\max _{x \in M}|\sigma|(x)$. Passing to a subsequence, we assume $p=\lim _{k \rightarrow+\infty} x_{k}$. Without loss of generality, we may assume blow-up occur, that is, $c_{k} \rightarrow+\infty$. Take a local coordinate system $(\Omega, x)$ around $p$. Using the idea in $[\underline{S}$, we define a sequence of functions,

$$
\varphi_{k}(x)=2 \alpha_{k}\left\langle\sigma_{k}\left(x_{k}\right), \sigma_{k}\left(x_{k}+r_{k} x\right)-\sigma_{k}\left(x_{k}\right)\right\rangle
$$

for some $r_{k}>0$, where $x \in \Omega_{k}$ with

$$
\Omega_{k}=\left\{x \in \mathbb{R}^{2} \mid x_{k}+r_{k} x \in \Omega\right\} .
$$

We then prove that, for suitable $r_{k}$,

$$
\varphi_{k} \rightarrow-2 \log \left(1+\pi|x|^{2}\right) \quad \text { in } \quad C_{\operatorname{loc}}^{2}\left(\mathbb{R}^{2}\right) .
$$

Then we prove that

$$
c_{k} \sigma_{k} \rightarrow G \quad \text { in } \quad C^{2}(\Omega), \quad \forall \Omega \subset \subset M \backslash\{p\},
$$

where $G$ is certain Green section. Finally, with the asymptotic behavior of $\sigma_{k}$ described above, we can establish the desired inequality and thus Theorem 1.1. In fact, we can give an upper bound of the functional $J_{4 \pi}$ in case that the blow-up happens.

Though we mainly follow the ideas in $[\mathrm{L}$ ) and $[\mathrm{L}-\mathrm{L}$, we should point out that, in this paper, the convergence of $c_{k} \sigma_{k}$ is derived differently from that of $[\mathrm{L}]$ or $[\mathrm{L}-\mathrm{L}]$ : The key gradient in $[\mathrm{L}]$ is the energy estimate

$$
\lim _{k \rightarrow+\infty} \int_{\left\{u_{k} \leq \frac{c_{k}}{A}\right\}}\left|\nabla u_{k}\right|^{2} d V_{g}=\frac{1}{A} .
$$

In this paper, though similar identity is used, the calculations are based on the local Pohozaev identities. We thank Professor Weiyue Ding who notice us possible application of the Pohozaev identity when we study the extremal function for Fontana's inequality on 4-dimensional manifold (see $[\mathrm{L}-\mathrm{Y}]$ ). Moreover, the method we get the upper bound of $J_{4 \pi}$ is also new: instead of capacity technique in $[\mathrm{L}$, we use a result of Carleson and Chang ([C-C]$)$ as follows:

Theorem $\mathbf{A}$ Let $B$ be unit ball in $\mathbb{R}^{2}$. Given any sequence $u_{k} \in H_{0}^{1,2}(B)$, if $u_{k} \rightarrow 0$, and $\int_{B_{\delta}}\left|\nabla u_{k}\right|^{2} d x \leq 1$, then we have

$$
\limsup _{k \rightarrow+\infty} \int_{B}\left(e^{4 \pi u_{k}^{2}}-1\right) d x \leq \pi e .
$$

The paper is organized as follows: In section 2, we settle some notations for use later. In section 3 we prove that $4 \pi$ is the best constant. Section 4 is blowing up analysis. We will prove the convergence of $c_{k} \sigma_{k}$ in section 5 . Then we finish the proof of our main theorem in section 6 . 
We hope our results can be a powerful tool in studying some problems arising from geometry and mathematical physics. In a forthcoming paper, we shall extend our results to high dimensional case and find some geometrical and physical applications.

\section{Preliminaries}

In this section, we clarify some notations. Take finite coordinate domains $\left\{\Omega_{k}\right\}$ which cover M. Let $\sigma$ be a smooth section of $E$, on each $\Omega_{k}$, we can set $\sigma=\sum_{i=1}^{n} u^{i} e_{i}$. We define

$$
\|\sigma\|_{H_{k}^{1,2}}^{2}=\int_{M} \sum_{i, j}\left(\left|\frac{\partial u^{i}}{\partial x^{j}}\right|^{2}+\sum_{k}\left|u^{i}\right|^{2}\right) d x
$$

and

$$
\|\sigma\|_{H^{1,2}(M, E)}=\sum_{k}\|\sigma\|_{H_{k}^{1,2}}
$$

Clearly, such norm is equivalent to

$$
\|\cdot\|=\left(\int_{M}\left(|\nabla \cdot|^{2}+|\cdot|^{2}\right) d V_{g}\right)^{\frac{1}{2}} .
$$

Let $\sigma$ be a parallel section, i.e. $\sigma \in H_{0}$. Then we have $\|\sigma\|_{H^{1,2}} \leq C\|\sigma\|_{L^{2}}$. By the compactness of Sobolev embedding from $H^{1,2}$ into $L^{2}$, we have

1. $H_{0}$ is a finite dimensional vector space. Throughout this paper, we use $\zeta_{1}, \cdots, \zeta_{m}$ to denote an orthogonal basis of $H_{0}$.

2. Poicaré inequality holds on $H_{1}$, i.e. for any $\sigma \in H_{1}$, we have

$$
\int_{M}|\sigma|^{2} d V_{g} \leq C \int_{M}\langle\nabla \sigma, \nabla \sigma\rangle d V_{g}
$$

then, on $H_{1}$, we can set

$$
\|\cdot\|_{H_{1}^{1,2}}=\left(\int_{M}\left(|\nabla \cdot|^{2}\right) d V_{g}\right)^{\frac{1}{2}}
$$

which is equivalent to the $H^{1,2}$ norm.

Throughout this paper, we will use $\left(\Omega_{p} ; x^{1}, x^{2}\right)$ to denote an isothermal coordinate system around $p$ with $p=(0,0)$. We can write the metric in such coordinate system as follows:

$$
g=e^{f_{p}}\left(d\left(x^{1}\right)^{2}+d\left(x^{2}\right)^{2}\right)
$$

with $f_{p}(0)=0$. Moreover, we always assume $e_{1}, e_{2}, \cdots, e_{n}$ to be an orthogonal basis of $E$ in $\Omega_{p}$.

We should explain some notations involving $\nabla \Phi$. Locally, when $\Phi=u^{k} e_{k}$ is a section of $E$, and $\nabla$ denotes the connection of $E$, then

$$
\langle\nabla \Phi, \nabla \Phi\rangle=e^{-f_{p}} \sum_{i=1,2}\left\langle\nabla_{\frac{\partial}{\partial x^{i}}} u^{k} e_{k}, \nabla_{\frac{\partial}{\partial x^{i}}} u^{k} e_{k}\right\rangle=\sum_{i=1,2}\left\langle\nabla_{\frac{\partial}{\partial x^{i}}} u^{k} e_{k}, \nabla_{\frac{\partial}{\partial x^{i}}} u^{k} e_{k}\right\rangle_{0} .
$$

When $\Phi$ is a function, then $\nabla \Phi$ is just the tangent vector $\frac{\partial \Phi}{\partial x^{1}} \frac{\partial}{\partial x^{1}}+\frac{\partial \Phi}{\partial x^{2}} \frac{\partial}{\partial x^{2}}$, and

$$
\left|\nabla_{0} \Phi\right|^{2}=\left|\frac{\partial \Phi}{\partial x^{1}}\right|^{2}+\left|\frac{\partial \Phi}{\partial x^{2}}\right|^{2}, \quad|\nabla \Phi|^{2}=e^{-f_{p}}\left|\nabla_{0} \Phi\right|^{2} .
$$


When $\Phi=\left(u^{1}, \cdots, u^{n}\right) \in H^{1,2}(\Omega) \times \cdots \times H^{1,2}(\Omega)$, we use $\left|\nabla_{0} \Phi\right|^{2}$ to denote

$$
\sum_{k=1}^{n}\left|\nabla_{0} u^{k}\right|^{2}
$$

Throughout this paper we use $B_{r}$ to denote the following open set of $\Omega_{p}$

$$
\left\{\left(x^{1}, x^{2}\right):\left|x^{1}\right|^{2}+\left|x^{2}\right|^{2}<r^{2}\right\} .
$$

Finally, we use $\Delta_{0}$ to denote the standard Laplacian on $\mathbb{R}^{2}$

$$
\frac{\partial^{2}}{\partial x^{1^{2}}}+\frac{\partial^{2}}{\partial x^{2}}
$$

\section{The best constants}

The main task of this section is to prove the following: $\sup _{\sigma \in \mathcal{H}_{1}} \int_{M} e^{\alpha|\sigma|^{2}} d V_{g}<+\infty$ for any $\alpha<4 \pi$, and $\sup _{\sigma \in \mathcal{H}_{1}} \int_{M} e^{\alpha|\sigma|^{2}} d V_{g}=+\infty$ for any $\alpha>4 \pi$. Firstly, we need a result in [Ch] (cf. Theorem 2.50 in $[\mathrm{Au}])$ :

Lemma 3.1. Let $B_{r}$ be a ball in $\mathbb{R}^{2}$, then for any $\beta<2 \pi$, we have

$$
\sup _{\int_{B_{r}}\left(|\nabla u|^{2}+u^{2}\right) d x=1} \int_{B_{r}} e^{\beta u^{2}} d x<C(r) .
$$

Then we have the following:

Corollary 3.2. Let $B_{r}$ be a ball in $\mathbb{R}^{2}$, and $u^{1}, \cdots, u^{n} \in H^{1,2}\left(B_{r}\right)$. Then we have

$$
\sup _{\sum_{i=1}^{n} \int_{B_{r}}\left(\left|\nabla u^{i}\right|^{2}+\left|u^{i}\right|^{2}\right) d x=1} \int_{B_{r}} e^{\beta \sum_{i=1}^{n}\left|u^{i}\right|^{2}} d x<C(r) .
$$

for any $\beta<2 \pi$.

Proof. We set $\lambda_{i}=\int_{M}\left(\left|\nabla u^{i}\right|^{2}+\left|u^{i}\right|^{2}\right) d x$, then $\int_{\Omega} e^{\beta \frac{\left|u^{i}\right|^{2}}{\lambda_{i}}} d x<C$. Hence

$$
\int_{\Omega} e^{\beta \sum_{i=1}^{n}\left|u^{i}\right|^{2}} d x \leq \prod_{i=1}^{n}\left(\int_{\Omega} e^{\beta \frac{\left|u^{i}\right|^{2}}{\lambda_{i}}} d x\right)^{\lambda_{i}} \leq C^{\sum_{i=1}^{n} \lambda_{i}}=C .
$$

Remark 3.1. Let $\Omega_{p}$ be a coordinate system around $p$, and $B_{r} \subset \Omega_{p}$. Given a section $\sigma=u^{k} e_{k}$, we set $U=\left(u^{1}, \cdots, u^{n}\right)$. Clearly, $\left|\nabla_{0} U\right|^{2}+|U|^{2} \leq C\left(\langle\nabla \sigma, \nabla \sigma\rangle+|\sigma|^{2}\right)$. Hence, for any $\alpha<\frac{2 \pi}{C}$, we have

$$
\sup _{\int_{B_{r}}\left(\langle\nabla \sigma, \nabla \sigma\rangle+|\sigma|^{2}\right) d V_{g}=1} \int_{B_{r}} e^{\alpha|\sigma|^{2}} d V_{g}<+\infty .
$$

As an consequence, we have the following lemma:

Lemma 3.3. There exists a positive number $\alpha$ such that $\sup _{\sigma \in \mathcal{H}_{1}} \int_{M} e^{\alpha|\sigma|^{2}} d V_{g}<+\infty$.

Denote $\tilde{\alpha}=\sup \left\{\alpha: \sup _{\sigma \in \mathcal{H}_{1}} \int_{M} e^{\alpha|\sigma|^{2}} d V_{g}<+\infty\right\}$. We shall prove that $\tilde{\alpha}=4 \pi$. 
Lemma 3.4. $\tilde{\alpha} \leq 4 \pi$.

Proof. Let $\Omega_{p}$ be a coordinate domain. By Moser's result [M], we can find a sequence $\left\{u_{k}\right\} \subset$ $H_{0}^{1,2}\left(B_{\frac{1}{k}}\right)$ such that $\int_{B_{\frac{1}{k}}}\left|\nabla_{0} u_{k}\right|^{2} d x=\int_{B_{\frac{1}{k}}}\left|\nabla u_{k}\right|^{2} d V_{g}=1$, and $\int_{B_{\frac{1}{k}}} e^{\left(4 \pi+\frac{1}{k}\right) u_{k}^{2}} d V_{g}>k$ as $k \rightarrow$ $+\infty$. We set

$$
\sigma_{k}=u_{k} e_{1}-\sum_{i=1}^{m}\left\langle u_{k} e_{1}, \zeta_{i}\right\rangle_{L^{2}} \zeta_{i} \in H_{1}
$$

Since $\left\|u_{k}\right\|_{L^{2}} \rightarrow 0$, we get

$$
\int_{M}\left\langle\nabla \sigma_{k}, \nabla \sigma_{k}\right\rangle d V_{g} \rightarrow 1, \text { and } \int_{M} e^{\left(4 \pi+\frac{1}{k}\right)\left|\sigma_{k}\right|^{2}} d V_{g} \rightarrow \infty
$$

Next, we prove an energy concentration phenomenon as follows:

Lemma 3.5. Let $\sigma_{k} \in \mathcal{H}_{1}$. If

$$
\lim _{k \rightarrow+\infty} \int_{M} e^{\alpha\left|\sigma_{k}\right|^{2}} d V_{g}=+\infty
$$

for all $\alpha>\tilde{\alpha}$, then passing to a subsequence, $\sigma_{k} \rightarrow 0$ in $H^{1,2}(M, E)$, and

$$
\left\langle\nabla \sigma_{k}, \nabla \sigma_{k}\right\rangle d V_{g} \rightarrow \delta_{p}
$$

for some $p \in M$.

Proof. Without loss of generality, we assume

$$
\begin{array}{lll}
\sigma_{k} \rightarrow \sigma_{0} & \text { weakly in } & H^{1,2}(M, E) \\
\sigma_{k} \rightarrow \sigma_{0} & \text { strongly in } & L^{2}(M, E) .
\end{array}
$$

We first claim that $\sigma_{0}=0$. Suppose not, we have

$$
\int_{M}\left\langle\nabla\left(\sigma_{k}-\sigma_{0}\right), \nabla\left(\sigma_{k}-\sigma_{0}\right)\right\rangle d V_{g} \rightarrow 1-\int_{M}\left\langle\nabla \sigma_{0}, \nabla \sigma_{0}\right\rangle d V_{g}<1
$$

Hence we can find a $\alpha_{1}>\tilde{\alpha}$ such that $\int_{M} e^{\alpha_{1}\left|\sigma_{k}\right|^{2}} d V_{g}$ is bounded, which contradicts (3.1).

Secondly we show the concentration phenomenon. Suppose

$$
\left\langle\nabla \sigma_{k}, \nabla \sigma_{k}\right\rangle d V_{g} \rightarrow \mu \neq \delta_{p}, \quad \forall p \in M
$$

Taking $\eta \in C_{0}^{\infty}\left(B_{\delta}(p)\right), \eta \equiv 1$ on $B_{\delta / 2}(p)$, one can easily see that

$$
\limsup _{k \rightarrow+\infty} \int_{B_{\delta}(p)}\left\langle\nabla\left(\eta \sigma_{k}\right), \nabla\left(\eta \sigma_{k}\right)\right\rangle d V_{g}<1 .
$$

Hence $e^{\left|\eta \sigma_{k}\right|^{2}}$ is bounded in $L^{\alpha}(M, E)$ for some $\alpha>\tilde{\alpha}$. A covering argument implies that $e^{\left|\sigma_{k}\right|^{2}}$ is bounded in $L^{\alpha}(M, E)$ for some $\alpha>\tilde{\alpha}$, which contradicts (3.1).

Corollary 3.6. $\quad \tilde{\alpha}=4 \pi$ 
Proof. By the definition of $\tilde{\alpha}$, we can find a sequence $\sigma_{k} \in \mathcal{H}_{1}$ such that

$$
\lim _{k \rightarrow+\infty} \int_{M} e^{\left(\tilde{\alpha}+\frac{1}{k}\right)\left|\sigma_{k}\right|^{2}} d V_{g}=+\infty .
$$

Then, applying Lemma 3.5, we get

$$
\left\langle\nabla \sigma_{k}, \nabla \sigma_{k}\right\rangle d V_{g} \rightarrow \delta_{p}, \text { and } \int_{M}\left|\sigma_{k}\right|^{2} d V_{g} \rightarrow 0 .
$$

Hence, for any $\eta$ which is 0 near $\mathrm{p}$, we have $\int\left(\left|\nabla\left(\eta \sigma_{k}\right)\right|^{2}+\left|\eta \sigma_{k}\right|^{2}\right) d V_{g} \rightarrow 0$. Applying Lemma 3.3 , one can find a subsequence (still denoted by $\sigma_{k}$ ) such that

$$
\lim _{k \rightarrow+\infty} \int_{\Omega} e^{q\left|\eta \sigma_{k}\right|^{2}} d V_{g}=\operatorname{Vol}(\Omega)
$$

for any $\Omega \subset \subset M \backslash\{p\}$ and $q>0$.

In coordinate around $p$, we set $\sigma_{k}=u_{k}^{i} e_{i}$. It is easy to check that

$$
\lim _{k \rightarrow+\infty} \int_{B_{2 r}} \sum_{i, j}\left|\frac{\partial\left(\eta^{\prime} u_{k}^{i}\right)}{\partial x^{j}}\right|^{2} d x=1,
$$

where $\eta^{\prime}$ is a cut-off function which is 1 on $B_{r}$. Then, similar to the proof of Corollary 3.2, we can deduce from Moser's result $[\mathrm{M}]$ that

$$
\lim _{k \rightarrow+\infty} \int_{B_{r}} e^{q\left|\sigma_{k}\right|^{2}} d x<+\infty
$$

for any $q<4 \pi$. Hence $\tilde{\alpha} \geq 4 \pi$, which together with Lemma 3.4 implies $\tilde{\alpha}=4 \pi$.

In a similar way, we can prove the following

Proposition 3.7 For any $\alpha<4 \pi$,

$$
\sup _{\sigma \in \mathcal{H}_{2}} \int_{M} e^{\alpha|\sigma|^{2}} d V_{g}<+\infty
$$

and for any $\alpha>4 \pi$,

$$
\sup _{\sigma \in \mathcal{H}_{2}} \int_{M} e^{\alpha|\sigma|^{2}} d V_{g}=+\infty
$$

\section{Blowing up analysis}

Let $\alpha_{k}$ be an increasing sequence which converges to $4 \pi$. In this section, we shall consider a sequence of sections $\sigma_{k}$ which attains $\sup _{\mathcal{H}_{1}} J_{\alpha_{k}}$, and analyze its blow-up behavior. First of all, we prove the following

Lemma 4.1. The functional $J_{\alpha_{k}}(\sigma)$ defined on the space $\mathcal{H}_{1}$ admits a smooth maximizer $\sigma_{k} \in \mathcal{H}_{1}$. Moreover, we have

$$
\lim _{k \rightarrow+\infty} \int_{M} e^{\alpha_{k}\left|\sigma_{k}\right|^{2}} d V_{g}=\sup _{\sigma \in \mathcal{H}_{1}} \int_{M} e^{4 \pi|\sigma|^{2}} d V_{g}
$$


Proof. It is easy to find $\sigma_{k} \in \mathcal{H}_{1}$ such that

$$
J_{\alpha_{k}}\left(\sigma_{k}\right)=\sup _{\sigma \in \mathcal{H}_{1}} J_{\alpha_{k}}(\sigma) .
$$

One can check that $\sigma_{k}$ satisfies the following Euler-Lagrange equation:

$$
-\Delta \sigma_{k}=\frac{\sigma_{k}}{\lambda_{k}} e^{\alpha_{k}\left|\sigma_{k}\right|^{2}}-\sum_{i=1}^{m} \gamma_{k}^{i} \zeta_{i}
$$

where $\Delta=\nabla^{*} \nabla$ is defined as follows: for any $\tau \in H^{1,2}(M, E)$

$$
\int_{M}\left\langle\nabla \sigma_{k}, \nabla \tau\right\rangle d V_{g}=\int_{M}\left\langle\Delta \sigma_{k}, \tau\right\rangle d V_{g} .
$$

It is easy to check that

$$
\lambda_{k}=\int_{M}\left|\sigma_{k}\right|^{2} e^{\alpha_{k}\left|\sigma_{k}\right|^{2}} d V_{g}, \quad \text { and } \gamma_{k}^{i}=\int_{M} \frac{\left\langle\sigma_{k}, \zeta_{i}\right\rangle}{\lambda_{k}} e^{\alpha_{k}\left|\sigma_{k}\right|^{2}} d V_{g} .
$$

Let $\Omega_{p}$ be an coordinate system around $p$. We set

$$
\sigma_{k}=u_{k}^{i} e_{i}, \quad \text { and } U_{k}=\left(u_{k}^{1}, u_{k}^{2} \cdots, u_{k}^{n}\right)
$$

and

$$
\nabla e_{j}=\Gamma_{j i}^{l} d x^{i} \otimes e_{l}
$$

Then, we get a local version of (4.2)

$$
-\Delta_{0} u_{k}^{j}=T_{s}^{j i} \frac{\partial u_{k}^{s}}{\partial x^{i}}+R_{i}^{j} u_{k}^{i}+e^{f_{p}\left(x_{k}+r_{k} x\right)}\left(\frac{u_{k}^{j}}{\lambda_{k}} e^{\alpha_{k}\left|\sigma_{k}\right|^{2}}-\sum_{i=1}^{m} \gamma_{k}^{i}\left\langle\zeta_{i}, e_{p}\right\rangle\right),
$$

where $T_{s}^{j i}$ and $R_{i}^{j}$ are smooth on $\Omega_{p}$. (4.4) can be written for simplicity

$$
-\Delta_{0} U_{k}=e^{f_{p}\left(x_{k}+r_{k} x\right)} \frac{U_{k}}{\lambda_{k}} e^{\alpha_{k}\left|U_{k}\right|^{2}}+T\left(\nabla U_{k}\right)+R\left(U_{k}\right)-\sum_{i} \gamma_{k}^{i}\left\langle U_{k}, \zeta_{i}\right\rangle .
$$

The standard elliptic estimates implies that $\sigma_{k} \in C^{\infty}(M)$.

Since for any fixed $\sigma \in \mathcal{H}$,

$$
\int_{M} e^{\alpha_{k}|\sigma|^{2}} d V_{g} \leq \int_{M} e^{\alpha_{k}\left|\sigma_{k}\right|^{2}} d V_{g}
$$

(4.1) follows immediately.

We assume that

$$
\lim _{k \rightarrow+\infty} \int_{M} e^{\alpha\left|\sigma_{k}\right|^{2}} d V_{g}=+\infty
$$

for all $\alpha>4 \pi$ (Otherwise, by the weakly compactness of $L^{p}$, passing to a subsequence, we have

$$
\lim _{k \rightarrow+\infty} \int_{M} e^{\alpha\left|\sigma_{k}\right|^{2}} d V_{g}=\int_{M} e^{\alpha\left|\sigma_{0}\right|^{2}} d V_{g}
$$


where $\sigma_{0}$ is the weak limit of $\sigma_{k}$. Hence, Theorem 1.1 holds). It follows from Lemma 3.5 that $\left\langle\nabla \sigma_{k}, \nabla \sigma_{k}\right\rangle d V_{g} \rightarrow \delta_{p}$. Given any $\Omega \subset \subset M \backslash\{p\}$, we take a cut-off function $\eta$ which is 0 at $p$, and 1 on $\Omega$, then

$$
\int_{M}\left\langle\nabla \eta \sigma_{k}, \nabla \eta \sigma_{k}\right\rangle d V_{g}=\int_{M} \eta^{2}\left\langle\nabla \sigma_{k}, \nabla \sigma_{k}\right\rangle+2 \eta\left\langle\sigma_{k} d \eta, \nabla \sigma_{k}\right\rangle d V_{g}+\int_{M}\left\langle\sigma_{k} d \eta, \sigma_{k} d \eta\right\rangle d V_{g} \rightarrow 0,
$$

hence $\int_{\Omega}\left(\left|\nabla \eta \sigma_{k}\right|^{2}+\left|\eta \sigma_{k}\right|^{2}\right) d V_{g} \rightarrow 0$. By Lemma 3.7, $e^{\alpha_{k}\left|\sigma_{k}\right|^{2}}$ is bounded in $L^{p}(\Omega)$ for any $p>0$. Standard elliptic estimates imply that $\sigma_{k} \rightarrow 0$ in $C^{\infty}(\Omega)$.

Lemma 4.2. Let $\frac{1}{\beta_{k}}=\int_{M} \frac{\left|\sigma_{k}\right|}{\lambda_{k}} e^{\alpha_{k}\left|\sigma_{k}\right|^{2}} d V_{g}$. Then, we have

$$
\liminf _{k \rightarrow+\infty} \beta_{k}=+\infty, \liminf _{k \rightarrow+\infty} \frac{\lambda_{k}}{\beta_{k}}=+\infty, \text { and } \limsup _{k \rightarrow+\infty} \gamma_{k}<+\infty \text {. }
$$

Proof. For any fixed $N>0$, we have

$$
\lim _{k \rightarrow+\infty} \int_{\left|\sigma_{k}\right| \leq N} e^{\alpha_{k}\left|\sigma_{k}\right|^{2}} d V_{g}=\int_{M} e^{0}=\operatorname{Vol}(M) .
$$

Then

$$
\lim _{k \rightarrow+\infty} \int_{M} e^{\alpha_{k}\left|\sigma_{k}\right|^{2}} \leq \operatorname{Vol}(M)+\lim _{k \rightarrow+\infty} \frac{1}{N^{2}} \int_{\left|\sigma_{k}\right|>N}\left|\sigma_{k}\right|^{2} e^{\alpha_{k}\left|\sigma_{k}\right|^{2}} d V_{g} \leq \operatorname{Vol}(M)+\lim _{k \rightarrow+\infty} \frac{\lambda_{k}}{N^{2}} .
$$

If $\lim _{k \rightarrow+\infty} \lambda_{k}<+\infty$, letting $N \rightarrow+\infty$, we get

$$
\int_{M} e^{\alpha_{k}\left|\sigma_{k}\right|^{2}} d V_{g} \leq \operatorname{Vol}(M)
$$

However, it follows from (4.1) that

$$
\lim _{k \rightarrow+\infty} \int_{M} e^{\alpha_{k}\left|\sigma_{k}\right|^{2}} d V_{g}=\sup _{\sigma \in \mathcal{H}_{1}} \int_{M} e^{4 \pi|\sigma|^{2}} d V_{g}>\operatorname{Vol}(M) .
$$

Therefore, $\liminf _{k \rightarrow+\infty} \lambda_{k}=+\infty$.

In the similar way, we have $\frac{\lambda_{k}}{\beta_{k}} \rightarrow+\infty$. Then, we have

$$
\left|\gamma_{k}^{i}\right| \leq c_{1}+c_{2} \int_{\left\{\left|\sigma_{k}\right|^{2} \geq 1\right\}} \frac{|\sigma|^{2}}{\lambda_{k}} e^{\alpha_{k}\left|\sigma_{k}\right|^{2}} d V_{g} \leq C
$$

for some constants $c_{1}, c_{2}$ and $C$ depending only on $M$. Moreover, we have

$$
\int_{M} \frac{\left|\sigma_{k}\right|}{\lambda_{k}} e^{\alpha_{k}\left|\sigma_{k}\right|^{2}} d V_{g} \leq \int_{M}\left(\frac{e^{4 \pi N^{2}}}{\lambda_{k}}+\frac{1}{\lambda_{k} N}|\sigma|^{2} e^{\alpha_{k}\left|\sigma_{k}\right|^{2}} d V_{g}\right)
$$

Letting $k \rightarrow+\infty$, and then $N \rightarrow+\infty$, we get $\frac{1}{\beta_{k}} \rightarrow 0$.

Let $c_{k}=\left|\sigma_{k}\right|\left(x_{k}\right)=\max _{x \in M}\left|\sigma_{k}\right|(x)$. By (4.4) and (4.5), $c_{k} \rightarrow+\infty$. Passing to a subsequence, we assume $x_{k} \rightarrow p$. Set $r_{k}^{2}=\lambda_{k} c_{k}^{-2} e^{-\alpha_{k} c_{k}^{2}}$. Since

we get

$$
1=\int_{M} \frac{\left|\sigma_{k}\right|^{2}}{\lambda_{k}} e^{\alpha_{k}\left|\sigma_{k}\right|^{2}} d V_{g} \leq \frac{c_{k}^{2}}{\lambda_{k}} e^{\frac{\alpha_{k}}{2} c_{k}^{2}} \int_{M} e^{\frac{\alpha_{k}}{2}\left|\sigma_{k}\right|^{2}} d V_{g} \leq C \frac{c_{k}^{2}}{\lambda_{k}} e^{\frac{\alpha_{k}}{2} c_{k}^{2}},
$$

$$
r_{k}^{2} e^{\frac{\alpha_{k}}{2} c_{k}^{2}} \rightarrow 0, \text { as } k \rightarrow+\infty .
$$


Let $\Omega_{p}$ be a local coordinate system around $p, \sigma_{k}=u_{k}^{i} e_{i}$ and $U_{k}=\left(u_{k}^{1}, \cdots, u_{k}^{n}\right)$. Denote

$$
V_{k}(x)=U_{k}\left(x_{k}+r_{k} x\right), \quad D_{k}=U_{k}\left(x_{k}\right)
$$

and

$$
C_{k}=\left(c_{k}^{1}, c_{k}^{2}, \cdots, c_{k}^{n}\right)=\frac{1}{\left|B_{1}\right|} \int_{B_{1}} V_{k} d x .
$$

A direct calculation shows

$$
-\Delta_{0}\left(V_{k}-C_{k}\right)=c_{k}^{-2} V_{k} e^{\alpha_{k}\left(\left|\sigma_{k}\right|^{2}-c_{k}^{2}\right)+f\left(x_{k}+r_{k} x\right)}+\mathcal{F}_{k} .
$$

where $\left\|\mathcal{F}_{k}\right\|_{L_{l o c}^{2}} \rightarrow 0$. Hence

$$
-\Delta_{0}\left(V_{k}-C_{k}\right) \rightarrow 0 \quad \text { in } \quad L_{l o c}^{2}\left(\mathbb{R}^{2}, \mathbb{R}^{n}\right), \quad \text { and } \int_{B_{L}}\left|\nabla\left(V_{k}-C_{k}\right)\right|^{2} d x \leq 1 .
$$

Then, by Poincare inequality, one has $\left\|V_{k}-C_{k}\right\|_{L^{2}\left(B_{L}\right)} \leq C(L)$ for any fixed $L>0$. By the standard elliptic estimates, $V_{k}-C_{k} \rightarrow V$ in $C_{l o c}^{0, \alpha}\left(\mathbb{R}^{2}, \mathbb{R}^{n}\right)$ for some $V \in H^{1,2}\left(\mathbb{R}^{2}, \mathbb{R}^{n}\right)$. Notice that $\int_{\mathbb{R}^{2}} \nabla_{0} V \nabla_{0} V^{T} d x \leq 1$, Liouville's theorem gives $V \equiv 0$. Furthermore we obtain

$$
V_{k}-D_{k}=\left(V_{k}-C_{k}\right)+\left(C_{k}-V_{k}(0)\right) \rightarrow 0 \text { in } C_{l o c}^{0, \alpha}\left(\mathbb{R}^{2}, \mathbb{R}^{n}\right) .
$$

Let

$$
w_{k}(x)=2 \alpha_{k} D_{k}\left(U_{k}\left(x_{k}+r_{k} x\right)-D_{k}\right)^{T} .
$$

Then we have

$$
-\Delta_{0} w_{k}=2 \alpha_{k} e^{f\left(x_{k}+r_{k} x\right)}\left(1+\frac{D_{k}\left(V_{k}-D_{k}\right)^{T}}{c_{k}^{2}}\right) e^{\alpha_{k}\left(\left|\sigma_{k}\right|^{2}\left(x_{k}+r_{k} x\right)-c_{k}^{2}\right)}+\mathcal{F}_{k}^{\prime},
$$

where $\left\|\mathcal{F}^{\prime}{ }_{k}\right\|_{L_{\text {loc }}^{2}} \rightarrow 0$. It is easy to see that on $B_{L}$

$$
\left(D_{k} U_{k}^{T}\right)\left(x_{k}+r_{k} x\right) \leq\left|D_{k}\right| \times\left|U_{k}^{T}\left(x_{k}+r_{k} x\right)\right| \leq c_{k}^{2}
$$

Hence

$$
w_{k}(x) \leq w_{k}(0)=0 .
$$

Then the identity

$$
\alpha_{k}\left(\left|\sigma_{k}\right|^{2}-c_{k}^{2}\right)=w_{k}+\alpha_{k}\left(V_{k}-D_{k}\right)\left(V_{k}-D_{k}\right)^{T},
$$

together with Harnack inequality and standard elliptic estimates, gives $w_{k} \rightarrow w$ in $C_{l o c}^{0, \alpha}\left(\mathbb{R}^{2}\right)$, where $w$ satisfies

$$
\left\{\begin{array}{l}
-\Delta_{0} w=8 \pi e^{w} \text { in } \mathbb{R}^{2} \\
w(0)=\sup _{\mathbb{R}^{2}} w=0 \\
\int_{\mathbb{R}^{2}} e^{w} d x \leq 1
\end{array}\right.
$$

By a result of $\left[\mathrm{C}-\mathrm{L}\right.$, we have $w(x)=-2 \log \left(1+\pi|x|^{2}\right)$ in $\mathbb{R}^{2}$ and $\int_{\mathbb{R}^{2}} e^{w}=1$.

In the rest of this section, we will discuss the convergence of $\beta_{k} \sigma_{k}$. 
Proposition 4.3. $\beta_{k} \sigma_{k} \rightarrow G$ weakly in $H^{1, q}(M, E)$ for any $1<q<2$, and $\beta_{k} \sigma_{k} \rightarrow G$ in $C^{2}(\Omega)$ for any $\Omega \subset \subset M \backslash\{p\}$, where $G \in C^{2}(M \backslash\{p\})$ satisfies

$$
-\Delta G=\theta \delta_{p}-\sum_{i=1}^{m}\left\langle\theta, \zeta_{i}\right\rangle \zeta_{i}
$$

for some $\theta \in E_{p}$.

Proof. Since $\sigma_{k} \rightarrow 0$ in $C^{\infty}\left(M \backslash B_{\delta}(p)\right)$, and $\frac{\beta_{k}}{\lambda_{k}} \rightarrow 0$, then for any $\varphi \in C^{\infty}(M, E)$, we have

$$
\int_{M \backslash B_{\delta}(p)} \frac{\beta_{k}\left\langle\varphi, \sigma_{k}\right\rangle}{\lambda_{k}} e^{\alpha_{k}\left|\sigma_{k}\right|^{2}} d V_{g} \rightarrow 0 .
$$

On $\Omega_{p}$, we set

$$
\theta^{i}=\lim _{k \rightarrow+\infty} \int_{\Omega_{p}} \frac{\beta_{k} u^{i}}{\lambda_{k}} e^{\alpha_{k}\left|\sigma_{k}\right|^{2}} d V_{g}
$$

and $\theta=\theta^{i} e_{i}$. It is easy to see that

$$
\int_{M} \frac{\left\langle\varphi, \sigma_{k}\right\rangle}{\lambda_{k}} e^{\alpha_{k}\left|\sigma_{k}\right|^{2}} d V_{g} \rightarrow\langle\varphi, \theta\rangle
$$

Moreover, we have

$$
\int_{M} \frac{\beta_{k}\left\langle\varphi, \sigma_{k}\right\rangle}{\lambda_{k}} e^{\alpha_{k}\left|\sigma_{k}\right|^{2}} d V_{g}=\int_{M} \beta_{k} \gamma_{k}^{i}\left\langle\varphi, \zeta_{i}\right\rangle d V_{g}
$$

for any $\varphi \in C^{\infty}(M, E)$. Then, we get

$$
\left|\int_{M} \beta_{k} \gamma_{k}^{i}\left\langle\varphi, \zeta_{i}\right\rangle d V_{g}\right| \leq\|\varphi\|_{C^{0}}
$$

therefore, $\sup _{k}\left|\beta_{k} \gamma_{k}\right|<+\infty$.

Let $m_{k}^{2}=\int_{M}\left|\beta_{k} \sigma_{k}\right|^{2} d V_{g}$. Firstly, we need to prove $\sup _{k} m_{k}<+\infty$. If $m_{k} \rightarrow+\infty$, then $\left\|\Delta \frac{\beta_{k} u_{k}}{m_{k}}\right\|_{L^{1}} \rightarrow 0$. It follows from Proposition 7.1 in the Appendix that

$$
\frac{\beta_{k} \sigma_{k}}{m_{k}} \rightarrow 0
$$

in $H^{1, p}$ for any $p<2$. Hence it follows from Poincaré inequality and compact embedding of Sobolev space that $\frac{\beta_{k} \sigma_{k}}{m_{k}} \rightarrow 0$ in $L^{2}$, which contradicts $\int_{M}\left|\frac{\beta_{k} \sigma_{k}}{m_{k}}\right|^{2} d V_{g}=1$.

Since $\sup _{k} m_{k}<+\infty$, applying Proposition 7.1 again, we get $\beta_{k} \sigma_{k}$ converges weakly in $H^{1, p}$ for any $p<2$. Therefore $\left\|\beta_{k} \sigma_{k}\right\|_{L^{q}} \leq C(q)$ for any $q>0$. Assume $\beta_{k} \sigma_{k} \rightarrow G$ weakly in $H^{1, q}(M, E)$. Then we have by Lemma 4.3 and standard elliptic estimate,

$$
\beta_{k} \sigma_{k} \rightarrow G \quad \text { in } \mathrm{C}^{2}(\Omega), \quad \forall \Omega \subset \subset \mathrm{M} \backslash\{\mathrm{p}\} .
$$

Testing the equation satisfied by $\beta_{k} \sigma_{k}$ with $\phi \in C^{\infty}(M, E)$, one has

$$
\begin{aligned}
\int_{M}\left\langle\nabla \phi, \nabla \beta_{k} \sigma_{k}\right\rangle d V_{g} & =\int_{M} \frac{\left\langle\phi, \beta_{k} \sigma_{k}\right\rangle}{\lambda_{k}} e^{\alpha_{k}\left|\sigma_{k}\right|^{2}} d V_{g}-\sum_{i=1}^{m} \int_{M}\left\langle\phi, \beta_{k} \gamma_{k}^{i} \zeta_{i}\right\rangle d V_{g} \\
& \rightarrow\langle\theta, \phi(p)\rangle-\sum_{i=1}^{m}\left\langle\theta, \zeta_{i}(p)\right\rangle \int_{M}\left\langle\phi, \zeta_{i}\right\rangle d V_{g}
\end{aligned}
$$

Hence

$$
\int_{M}\langle\nabla \phi, \nabla G\rangle d V_{g}=\langle\theta, \phi\rangle(p)-\sum_{i=1}^{m}\left\langle\theta, \zeta_{i}(p)\right\rangle \int_{M}\left\langle\phi, \zeta_{i}\right\rangle d V_{g}
$$


and whence (4.9) holds. This completes the proof of the lemma.

\section{Applications of Pohozaev identity}

In this section, we will calculate $\lim _{k \rightarrow+\infty} \int_{M} e^{\alpha_{k}\left|\sigma_{k}\right|^{2}} d V_{g}$. The key gradient we use is Pohozaev identity. Let $B_{\delta}(p) \subset \Omega_{p}$. Testing equation (4.2) with $\eta(r) r \nabla_{\frac{\partial}{\partial r}} \sigma_{k}$, we have

$\int_{B_{\delta}(p)}\left\langle\nabla \eta(r) r \nabla_{\frac{\partial}{\partial r}} \sigma_{k}, \nabla \sigma_{k}\right\rangle d V_{g}=\int_{B_{\delta}(p)}\left\langle\eta(r) r \nabla_{\frac{\partial}{\partial r}} \sigma_{k}, \sigma_{k}\right\rangle \frac{e^{\alpha_{k}\left|\sigma_{k}\right|^{2}}}{\lambda_{k}} d x+\int_{B_{\delta}(p)} \gamma_{k}^{i}\left\langle\eta(r) r \nabla_{\frac{\partial}{\partial r}} \sigma_{k}, \zeta_{i}\right\rangle d V_{g}$,

where $\eta$ is a cut-off function which is 0 outside $\partial B_{\delta}$ and 1 on $B_{\frac{\delta}{2}}, r=\sqrt{\left(x^{1}\right)^{2}+\left(x^{2}\right)^{2}}$.

$$
\begin{aligned}
\left\langle\nabla \eta(r) r \nabla_{\frac{\partial}{\partial r}} \sigma_{k}, \nabla \sigma_{k}\right\rangle_{0}= & \sum_{j}\left\langle\nabla_{j} \eta(r) x^{p} \nabla_{p} \sigma_{k}, \nabla_{j} \sigma_{k}\right\rangle_{0} \\
= & \eta(r)\left\langle\nabla \sigma_{k}, \nabla \sigma_{k}\right\rangle_{0}+\eta^{\prime}(r) \frac{x^{i} x^{j}}{r}\left\langle\nabla_{i} \sigma_{k}, \nabla_{j} \sigma_{k}\right\rangle_{0}+\eta(r)\left\langle x^{i} \nabla_{j} \nabla_{i} \sigma_{k}, \nabla_{j} \sigma_{k}\right\rangle_{0} \\
= & \eta(r)\left\langle\nabla \sigma_{k}, \nabla \sigma_{k}\right\rangle_{0}+\eta^{\prime}(r) \frac{x^{i} x^{j}}{r}\left\langle\nabla_{i} \sigma_{k}, \nabla_{j} \sigma_{k}\right\rangle_{0}+\eta(r)\left\langle x^{i} \nabla_{i} \nabla_{j} \sigma_{k}, \nabla_{j} \sigma_{k}\right\rangle_{0} \\
& +\eta(r) \sum_{i, j}\left\langle x^{i} R_{i j} \sigma_{k}, \nabla_{j} \sigma_{k}\right\rangle_{0} \\
= & \eta(r)\left\langle\nabla \sigma_{k}, \nabla \sigma_{k}\right\rangle_{0}+\eta^{\prime}(r) \frac{x^{i} x^{j}}{r}\left\langle\nabla_{i} \sigma_{k}, \nabla_{j} \sigma_{k}\right\rangle_{0}+\frac{1}{2} \eta(r) r \frac{\partial}{\partial r}\left\langle\nabla \sigma_{k}, \sigma_{k}\right\rangle_{0} \\
& +\eta(r) \sum_{i, j}\left\langle x^{i} R_{i j} \sigma_{k}, \nabla_{j} \sigma_{k}\right\rangle_{0}+\eta(r) S_{0}\left(\nabla \sigma_{k}, \nabla \sigma_{k}\right),
\end{aligned}
$$

where $S_{0}=e^{-f} S$, and

$$
S\left(\sigma_{1}, \sigma_{2}\right)=\frac{1}{2}\left(r \frac{\partial}{\partial r}\left\langle\sigma_{1}, \sigma_{2}\right\rangle-\left\langle r \nabla_{\frac{\partial}{\partial r}} \sigma_{1}, \sigma_{2}\right\rangle-\left\langle\sigma_{1}, r \nabla_{\frac{\partial}{\partial r}} \nabla \sigma_{2}\right\rangle\right) .
$$

Since we do not assume $\nabla h=0, S \neq 0$ generally. We set

$$
\eta(r)= \begin{cases}1 & r \leq \delta \\ \frac{r-\delta-a}{-a} & \delta \leq r \leq \delta+a \\ 0 & r \geq \delta+a\end{cases}
$$

Letting $a \rightarrow 0$, we get

$$
\begin{aligned}
\int_{B_{\delta}(p)}\left\langle\nabla r \nabla_{\frac{\partial}{\partial r}} \sigma_{k}, \nabla \sigma_{k}\right\rangle_{0} d x= & \int_{\Omega_{p}}\left(\left\langle\nabla \sigma_{k}, \nabla \sigma_{k}\right\rangle_{0}+\frac{1}{2} r \frac{\partial}{\partial r}\left\langle\nabla \sigma_{k}, \sigma_{k}\right\rangle_{0}+\sum_{i, j}\left\langle x^{i} R_{i j} \sigma_{k}, \nabla_{j} \sigma_{k}\right\rangle_{0}\right) d x \\
& -\int_{\partial B_{\delta}(p)} \frac{x^{i} x^{j}}{r}\left\langle\nabla_{i} \sigma_{k}, \nabla_{j} \sigma_{k}\right\rangle_{0} d S+\int_{B_{\delta}(p)} S\left(\nabla \sigma_{k}, \nabla \sigma_{k}\right) d V_{g} .
\end{aligned}
$$

Clearly,

$$
\begin{aligned}
\int_{B_{\delta}(p)} \frac{1}{2} r \frac{\partial}{\partial r}\left\langle\nabla \sigma_{k}, \sigma_{k}\right\rangle_{0} d x & =\int_{0}^{\delta} \pi r^{2} \frac{\partial}{\partial r}\left(\int_{S^{1}}\left\langle\nabla \sigma_{k}, \nabla \sigma_{k}\right\rangle_{0} d \theta\right) d r \\
& =\frac{1}{2} \int_{\partial B_{\delta}(p)} r\left\langle\nabla \sigma_{k}, \nabla \sigma_{j}\right\rangle_{0} d S-\int_{B_{\delta}(p)}\left\langle\sigma_{k}, \sigma_{k}\right\rangle_{0} d x
\end{aligned}
$$


Then

$$
\begin{aligned}
\int_{B_{\delta}(p)}\left\langle\nabla r \nabla_{\frac{\partial}{\partial r}} \sigma_{k}, \nabla \sigma_{k}\right\rangle_{0} d x= & \int_{\Omega_{p}} \sum_{i, j}\left\langle x^{i} R_{i j} \sigma_{k}, \nabla_{j} \sigma_{k}\right\rangle_{0} d x-\int_{\partial B_{\delta}(p)} \frac{x^{i} x^{j}}{r}\left\langle\nabla_{i} \sigma_{k}, \nabla_{j} \sigma_{k}\right\rangle_{0} d S \\
& +\frac{1}{2} \int_{\partial B_{\delta}(p)} r\left\langle\nabla \sigma_{k}, \nabla \sigma_{j}\right\rangle_{0} d S+\int_{B_{\delta}(p)} S\left(\nabla \sigma_{k}, \nabla \sigma_{k}\right) d V_{g} .
\end{aligned}
$$

On the other hand, we have

$$
\int_{B_{\delta}} e^{-f}\left\langle r \nabla_{\frac{\partial}{\partial r}} \sigma_{k}, \sigma_{k}\right\rangle_{0} \frac{e^{\alpha_{k}\left|\sigma_{k}\right|^{2}}}{\lambda_{k}} d x=\int_{B_{\delta}} e^{-f} r \frac{\partial}{\partial r}\left\langle\sigma_{k}, \sigma_{k}\right\rangle_{0} \frac{e^{\alpha_{k}\left|\sigma_{k}\right|^{2}}}{\lambda_{k}} d x+\int_{B_{\delta}} \frac{S(\sigma, \sigma)}{\lambda_{k}} e^{\alpha_{k}\left|\sigma_{k}\right|^{2}} d V_{g}
$$

and

$$
\begin{aligned}
\int_{B_{\delta}} e^{-f}\left\langle r \nabla_{\frac{\partial}{\partial r}} \sigma_{k}, \sigma_{k}\right\rangle_{0} \frac{e^{\alpha_{k}\left|\sigma_{k}\right|^{2}}}{\lambda_{k}} d x= & \int_{0}^{\delta} 2 \pi r^{2} \frac{\partial}{\partial r}\left(\int_{S^{1}} \frac{e^{\alpha_{k}|\sigma|^{2}}}{2 \alpha_{k} \lambda_{k}} e^{-f} d \theta\right) d r+\int_{B_{\delta}} \frac{e^{\alpha_{k}|\sigma|^{2}}}{\alpha_{k} \lambda_{k}} r \frac{\partial f}{\partial r} d V_{g} . \\
= & \int_{\partial B_{\delta}(p)} r \frac{e^{\alpha_{k}\left|\sigma_{k}\right|^{2}}}{\alpha_{k} \lambda_{k}} d S_{g}-\int_{B_{\delta}(p)} \frac{e^{\alpha_{k}\left|\sigma_{k}\right|^{2}}}{\alpha_{k} \lambda_{k}} d V_{g} \\
& +\int_{B_{\delta}} \frac{e^{\alpha_{k}|\sigma|^{2}}}{\alpha_{k} \lambda_{k}} r \frac{\partial f}{\partial r} d V_{g} .
\end{aligned}
$$

Therefore, we get

$$
\begin{aligned}
\int_{B_{\delta}(p)}(1+O(r)) \frac{e^{\alpha_{k}\left|\sigma_{k}\right|^{2}}}{\alpha_{k} \lambda_{k}} d V_{g}= & \int_{\partial B_{\delta}(p)} r \frac{e^{\alpha_{k}\left|\sigma_{k}\right|^{2}}}{\alpha_{k} \lambda_{k}} d S_{g}-\int_{B_{\delta}(p)} \sum_{i, j}\left\langle x^{i} R_{i j} \sigma_{k}, \nabla_{j} \sigma_{k}\right\rangle_{0} d x \\
& -\int_{\partial B_{\delta}(p)} \frac{x^{i} x^{j}}{r}\left\langle\nabla_{i} \sigma_{k}, \nabla_{j} \sigma_{k}\right\rangle_{0} d S-\frac{1}{2} \int_{\partial B_{\delta}(p)} r\left\langle\nabla \sigma_{k}, \nabla \sigma_{k}\right\rangle_{0} d S \\
& +\int_{B_{\delta}} \gamma_{k}^{i}\left\langle\eta(r) r \nabla_{\frac{\partial}{\partial r}} \sigma_{k}, \zeta_{i}\right\rangle d V_{g} \\
& +\int_{B_{\delta}}\left(S\left(\nabla \sigma_{k}, \nabla \sigma_{k}\right)-\frac{S\left(\sigma_{k}, \sigma_{k}\right)}{\lambda_{k}} e^{\alpha_{k}\left|\sigma_{k}\right|^{2}}\right) d V_{g} .
\end{aligned}
$$

Firstly, letting $k \rightarrow+\infty$, we have

$$
\int_{B_{\delta}} \gamma_{k}^{i}\left\langle\eta(r) r \nabla_{\frac{\partial}{\partial r}} \beta_{k} \sigma_{k}, \zeta_{i}\right\rangle d V_{g} \leq C\left\|\nabla \beta_{k} \sigma_{k}\right\|_{H_{B_{\delta}}^{1, q}} \rightarrow C\|\nabla G\|_{H^{1, q}\left(B_{\delta}\right)}
$$

and

$\int_{B_{\delta}(p)} \sum_{i, j}\left(\left\langle x^{i} R_{i j} \beta_{k} \sigma_{k}, \nabla_{j} \beta_{k} \sigma_{k}\right\rangle_{0}+S\right) d x \leq C\left\|\beta_{k} \sigma_{k}\right\|_{L^{q^{\prime}}\left(B_{\delta}\right)}|| \nabla \beta_{k} \sigma_{k}\left\|_{L^{q}\left(B_{\delta}\right)} \rightarrow C\right\| G\left\|_{L^{q^{\prime}\left(B_{\delta}\right)}}\right\| \nabla G \|_{L^{q}\left(B_{\delta}\right)}$, where $1<q<2$. Secondly, applying Lemma 7.2 in the Appendix, we get

$$
\begin{aligned}
& \int_{\partial B_{\delta}(p)} \frac{x^{i} x^{j}}{r}\left\langle\nabla_{i} \beta_{k} \sigma_{k}, \nabla_{j} \beta_{k} \sigma_{k}\right\rangle_{0} d S_{0}+\frac{1}{2} \int_{\partial B_{\delta}(p)} r\left\langle\nabla \beta_{k} \sigma_{k}, \nabla \beta_{k} \sigma_{j}\right\rangle_{0} d S \\
& \rightarrow \int_{\partial B_{\delta}(p)} \frac{x^{i} x^{j}}{r}\left\langle\nabla_{i} G, \nabla_{j} G\right\rangle_{0} d S-\frac{1}{2} \int_{\partial B_{\delta}(p)}^{r} r\langle\nabla G, \nabla G\rangle_{0} d S \\
& =\frac{1}{4 \pi}+O\left(\delta^{\gamma}\right) .
\end{aligned}
$$

Thirdly, since $\sigma_{k} \rightarrow 0$ in $\partial B_{\delta}$, we get

$$
\int_{\partial B_{\delta}(p)} \frac{e^{\alpha_{k}\left|\sigma_{k}\right|^{2}}}{\alpha_{k}} \rightarrow \frac{\left|\partial B_{\delta}\right|}{4 \pi}
$$


Finally, we set $S\left(\sigma_{1}, \sigma_{2}\right)=\left\langle A \sigma_{1}, \sigma_{2}\right\rangle$, where $\|A\|_{C^{0}}=O(r)$. Take a cut-off function $\eta$ which is 0 outsider $B_{2 \delta}$ and 1 in $B_{\delta}$ with $|\nabla \eta|<\frac{1}{\delta}$. We have

$$
\begin{aligned}
\int_{B_{2 \delta}(p)}\left\langle A \nabla \eta \sigma_{k}, \nabla \sigma_{k}\right\rangle & =\int_{B_{2 \delta}(p)}\left\langle\nabla A \eta \sigma_{k}, \nabla \sigma_{k}\right\rangle d V_{g}-\int_{B_{2 \delta}(p)}\left\langle(\nabla A) \sigma_{k}, \nabla \sigma_{k}\right\rangle d V_{g} \\
& =\int_{B_{2 \delta}(p)} \frac{\left\langle A \eta \sigma_{k}, \sigma_{k}\right\rangle}{\lambda_{k}} e^{\alpha_{k}\left|\sigma_{k}\right|^{2}} d V_{g}-\int_{B_{2 \delta}(p)}\left\langle(\nabla A) \sigma_{k}, \nabla \sigma_{k}\right\rangle d V_{g}
\end{aligned}
$$

Hence, we get

$$
\begin{aligned}
\beta_{k}^{2} \int_{B_{\delta}} & \left(S\left(\nabla \sigma_{k}, \nabla \sigma_{k}\right)-S\left(\sigma_{k}, \sigma_{k}\right) \frac{e^{\alpha_{k}\left|\sigma_{k}\right|^{2}}}{\lambda_{k}}\right) d V_{g} \\
\rightarrow & \rightarrow(1) \int_{B_{2 \delta}(p)}\langle(\nabla A) \eta G, \nabla G\rangle d V_{g}+O(\delta) \int_{B_{2 \delta}(p) \backslash B_{\delta}(p)} \eta|\nabla G|^{2} d V_{g} .
\end{aligned}
$$

So, we get

$$
\lim _{k \rightarrow+\infty} \int_{B_{\delta}(p)}(1+O(r)) e^{\alpha_{k}\left|\sigma_{k}\right|^{2}} d V_{g}=\frac{\left|\partial B_{\delta}\right|}{4 \pi}+\lim _{k \rightarrow+\infty} \frac{\lambda_{k}}{\beta_{k}^{2}}+h(\delta),
$$

where $\lim _{\delta \rightarrow 0} h(\delta)=0$. Clearly, $\lim _{k \rightarrow+\infty} \int_{M \backslash B_{\delta}(p)} e^{\alpha_{k}\left|\sigma_{k}\right|^{2}} d V_{g}=\left|M \backslash B_{\delta}(p)\right|$, we get

$$
\lim _{k \rightarrow+\infty} \int_{M} e^{\alpha_{k}\left|\sigma_{k}\right|^{2}} d V_{g}=\operatorname{Vol}(M)+\lim _{k \rightarrow+\infty} \frac{\lambda_{k}}{\beta_{k}^{2}} .
$$

The following lemma is very important:

Lemma 5.1. we have $\frac{c_{k}}{\beta_{k}} \rightarrow 1$.

Proof. Since

we have $c_{k} \geq \beta_{k}$.

$$
\frac{c_{k}}{\beta_{k}}=\int_{M} \frac{c_{k} \sigma_{k}}{\lambda_{k}} e^{\alpha_{k}\left|\sigma_{k}\right|^{2}} \geq \int_{M} \frac{\left|\sigma_{k}\right|^{2}}{\lambda_{k}} e^{\alpha_{k}\left|\sigma_{k}\right|^{2}} d V_{g}
$$

Assume the lemma is not true, then we can find $A>1$ such that $\frac{c_{k}}{A}>A \beta_{k}$ for sufficiently large $k$. Denote

$$
\tau=\lim _{k \rightarrow+\infty} \frac{\sigma_{k}}{c_{k}}=\tau^{i} e_{i}
$$

Without loss of generality, we assume $\tau^{1}>0$. By the equation (4.4), we have for any fixed $L>0$,

$$
\begin{aligned}
\int_{M}\left|\nabla_{0} \eta\left(u_{k}^{i}-\frac{u_{k}^{i}\left(x_{k}\right)}{A}\right)^{+}\right|^{2} d x & =\int_{\Omega_{p}} \nabla_{0} u_{k}^{i} \nabla_{0} \eta\left(u_{k}^{i}-\frac{u_{k}^{i}\left(x_{k}\right)}{A}\right)^{+} d x+o_{k}(1) \\
& =\int_{\Omega_{p}} \eta\left(u_{k}^{i}-\frac{u_{k}^{i}\left(x_{k}\right)}{A}\right)^{+} \frac{u_{k}^{i}}{\lambda_{k}} e^{\alpha_{k}\left|\sigma_{k}\right|^{2}} d V_{g}+o_{k}(1) \\
& \geq \int_{B_{L r_{k}\left(x_{k}\right)}} \frac{\left(u_{k}^{i}-\frac{u_{k}^{i}\left(x_{k}\right)}{A}\right) u_{k}^{i}}{\lambda_{k}} e^{\alpha_{k}\left|\sigma_{k}\right|^{2}} d V_{g}+o_{k}(1) \\
& =\int_{B_{L}(0)}\left(\frac{u_{k}^{i}\left(x_{k}\right)(A-1)+o_{k}(1)}{A}\right) \frac{u_{k}^{i}}{c_{k}^{2}} e^{\varphi_{k}(x)+o_{k}(1)} d x+o_{k}(1) .
\end{aligned}
$$

Letting $k \rightarrow+\infty$, then $L \rightarrow+\infty$, we get

$$
\liminf _{k \rightarrow+\infty} \int_{M}\left|\nabla \eta\left(u_{k}^{1}-\frac{u_{k}^{1}\left(x_{k}\right)}{A}\right)^{+}\right|^{2} d V_{g} \geq\left(1-\frac{1}{A}\right) \tau^{1^{2}} .
$$


Let $\sigma_{k}^{A}=\eta u_{k}^{A, i} e_{i}=\eta\left(\min \left\{u_{k}^{1}(x), \frac{u_{k}\left(x_{k}\right)}{A}\right\} e_{1}+\sum_{i=2}^{n} u_{k}^{i} e_{i}\right)$, where $\eta$ is a cut-off function which is 0 $M \backslash B_{2 \delta}(p)$, and 1 in $B_{\delta}(p)$. Since

$$
\left|\nabla \sigma_{k}^{A}\right|^{2}=\eta \sum_{i=1}\left|\nabla_{0} u_{k}^{A, i}\right|^{2}+O\left(\left|\sigma_{k}^{A}\right|\left|\nabla \sigma_{k}^{A}\right|\right)+O\left(\left|\sigma_{k}^{A}\right|^{2}\right),
$$

we get

$$
\limsup _{k \rightarrow+\infty} \int_{M}\left(\left|\nabla \sigma_{k}^{A}\right|^{2}+\left|\sigma_{k}^{A}\right|^{2}\right) d V_{g} \leq 1-\frac{A-1}{A} \tau^{1^{2}}<1 .
$$

Then, by Proposition 3.7, $e^{\alpha_{k}\left|\sigma_{k}^{A}\right|}$ is bounded in $L^{q}$ for some $q>1$. Since

$\int_{\Omega_{p} \cap\left\{u_{k}^{1} \leq \frac{\tau^{1} c_{k}}{A}\right\}} \frac{\beta_{k}}{\lambda_{k}}\left|\sigma_{k}\right| e^{\alpha_{k}\left|\sigma_{k}\right|^{2}} d V_{g} \leq \frac{\beta_{k}}{\lambda_{k}} \int_{\Omega_{p}}\left|\sigma_{k}\right| e^{\alpha_{k}\left|\sigma_{k}^{A}\right|^{2}} d V_{g} \leq \frac{\beta_{k}}{\lambda_{k}}\left\|\sigma_{k}\right\|_{L^{q^{\prime}}(M)}|| e^{\alpha_{k}\left|\sigma_{k}^{A}\right|^{2}} \|_{L^{q}(M)} \rightarrow 0$.

In the same way, for any $\tau^{i}$, we have

$$
\int_{\Omega_{p} \cap\left\{\left|u_{k}^{i}\right| \leq \frac{\tau^{i} c_{k}}{A}\right\}} \frac{\beta_{k}}{\lambda_{k}}\left|\sigma_{k}\right| e^{\alpha_{k}\left|\sigma_{k}\right|^{2}} d V_{g} \rightarrow 0
$$

Since $|\tau|=1$, we obtain

$$
\begin{aligned}
1 & \geq \lim _{k \rightarrow+\infty} \int_{\left\{\left|\sigma_{k}\right| \geq A \beta_{k}\right\}} \frac{\left|\sigma_{k}\right|^{2}}{\lambda_{k}} e^{\alpha_{k}\left|\sigma_{k}\right|^{2}} d V_{g} \\
& \geq A \lim _{k \rightarrow+\infty} \beta_{k} \int_{\left\{x \in \Omega_{p}:\left|u_{k}^{i}\right| \geq A \tau^{i} \beta_{k}, \forall i\right\}} \frac{\left|\sigma_{k}\right|}{\lambda_{k}} e^{\alpha_{k}\left|\sigma_{k}\right|^{2}} d V_{g} \\
& \geq A \lim _{k \rightarrow+\infty} \beta_{k} \int_{M} \frac{\left|\sigma_{k}\right|}{\lambda_{k}} e^{\alpha_{k}\left|\sigma_{k}\right|^{2}} d V_{g}-A \lim _{k \rightarrow+\infty} \sum_{i} \int_{\left\{\left|u_{k}^{i}\right| \leq \frac{\tau^{i} c_{k}}{A}\right\}} \frac{\beta_{k}\left|\sigma_{k}\right|}{\lambda_{k}} e^{\alpha_{k}\left|\sigma_{k}\right|^{2}} d V_{g} \\
& =A .
\end{aligned}
$$

This contradict with the choice of $A$.

Corollary 5.2. We have $\tau=\theta$, and

$$
\lim _{k \rightarrow+\infty} \int_{M}\left(e^{\alpha_{k}\left|\sigma_{k}\right|^{2}}-1\right) d V_{g}=\lim _{L \rightarrow+\infty} \lim _{k \rightarrow+\infty} \int_{B_{L r_{k}}\left(x_{k}\right)} e^{\alpha_{k}\left|\sigma_{k}\right|^{2}} d V_{g} .
$$

Proof. By a straightforward calculation, we have

$$
\int_{B_{L r_{k}}\left(x_{k}\right)} e^{\alpha_{k}\left|\sigma_{k}\right|^{2}} d V_{g}=\frac{\lambda_{k}}{c_{k}^{2}} \int_{B_{L}} e^{w} d x(1+o(1)) .
$$

The above inequality together with (5.1) implies (5.2).

It is not difficult to check that

$$
\lim _{L \rightarrow+\infty} \lim _{k \rightarrow+\infty} \int_{B_{L r_{k}}\left(x_{k}\right)} \frac{\beta_{k}\left|\sigma_{k}\right|}{\lambda_{k}} e^{\alpha_{k}\left|\sigma_{k}\right|^{2}} d V_{g}=1,
$$

and

$$
\lim _{L \rightarrow+\infty} \lim _{k \rightarrow+\infty} \int_{B_{L r_{k}}\left(x_{k}\right)} \frac{\beta_{k} u_{k}^{i}}{\lambda_{k}} e^{\alpha_{k}\left|\sigma_{k}\right|^{2}} d V_{g}=\tau^{i}
$$


Hence

$$
\left|\theta^{i}-\tau^{i}\right|=\lim _{L \rightarrow+\infty} \lim _{k \rightarrow+\infty} \int_{\Omega_{p} \backslash B_{L r_{k}}\left(x_{k}\right)} \frac{\beta_{k} u_{k}^{i}}{\lambda_{k}} e^{\alpha_{k}\left|\sigma_{k}\right|^{2}} d V_{g} \leq 1-\lim _{L \rightarrow+\infty} \lim _{k \rightarrow+\infty} \int_{B_{L r_{k}}\left(x_{k}\right)} \frac{\beta_{k}\left|\sigma_{k}\right|}{\lambda_{k}} e^{\alpha_{k}\left|\sigma_{k}\right|^{2}} d V_{g}=0 .
$$

\section{The proof of theorem $\mathbf{1 . 1}$}

On $\Omega_{p}$, we set $\tilde{U}_{k}=\left(\left|\tilde{u}_{k}^{1}\right|, \cdots,\left|\tilde{u}_{k}^{n}\right|\right)$. The following lemma is very important for the rest of our arguments:

Lemma 6.1. We have

$$
\int_{B_{\delta}(p)}\left|\nabla_{0} \tilde{U}_{k}\right|^{2} d x \leq \int_{B_{\delta}(p)}\left\langle\nabla \sigma_{k}, \nabla \sigma_{k}\right\rangle d V_{g}+\frac{\rho(\delta)}{c_{k}^{2}},
$$

where $\rho(\delta)$ is a continuous function of $\delta$ with $\rho(0)=0$.

Proof. It is well-known that

$$
\int_{B_{\delta}(p)}\left|\nabla_{0} \tilde{U}_{k}\right|^{2} d x=\int_{B_{\delta}(p)}\left|\nabla_{0} U_{k}\right|^{2} d x
$$

We set

$$
\nabla \sigma_{k}=\left(\frac{\partial u_{k}^{p}}{\partial x^{1}}+\frac{\partial u_{k}^{p}}{\partial x^{2}}\right) e_{p}+A_{i}^{p} u_{k}^{i} e_{p}
$$

where $A^{p}$ 's are smooth functions. Hence, we get

$$
\left|\nabla_{0} U_{k}\right|^{2}=\left|\nabla_{0} U_{k}\right|^{2}-2\left\langle A\left(\sigma_{k}\right), \nabla \sigma_{k}\right\rangle+\left|A U_{k}\right|^{2} .
$$

Since

we get

$$
\begin{aligned}
\int_{B_{\delta}(p)} c_{k}^{2}\left\langle A\left(\sigma_{k}\right), \nabla \sigma_{k}\right\rangle d x & \leq C\left(\int_{B_{\delta}(p)}\left|A\left(c_{k} \sigma_{k}\right)\right|^{q} d x\right)^{\frac{1}{q}}\left(\int_{B_{\delta}(p)}\left|\nabla c_{k} \sigma_{k}\right|^{p} d x\right)^{\frac{1}{p}} \\
& \leq C\left(\int_{B_{\delta}(p)}\left|c_{k} \sigma_{k}\right|^{q} d x\right)^{\frac{1}{q}}\left(\int_{B_{\delta}(p)}\left|\nabla c_{k} \sigma_{k}\right|^{p} d x\right)^{\frac{1}{p}} \\
& \rightarrow C\left(\int_{B_{\delta}(p)}|G|^{q} d x\right)^{\frac{1}{q}}\left(\int_{B_{\delta}(p)}|\nabla G|^{p} d x\right)^{\frac{1}{p}}
\end{aligned}
$$

Similarly we have

$$
\int_{B_{\delta}(p)}\left\langle A\left(\sigma_{k}\right), \nabla \sigma_{k}\right\rangle d V_{g} \leq \frac{\rho_{1}(\delta)}{c_{k}^{2}}
$$

$$
\int_{B_{\delta}(p)}\left|A\left(\sigma_{k}\right)\right|^{2} \leq \frac{\rho_{2}(\delta)}{c_{k}^{2}}
$$

The lemma follows immediately from the above two inequalities.

On $\Omega_{p}$, we can write $G$ as follows :

$$
G=-\frac{\log r}{2 \pi} \tau+s_{p}+\theta .
$$

where $s_{p}$ is a constant section, and $\theta$ is continuous local section of $E$ with $\theta(0)=0$. Then Theorem 1.1 follows from the following proposition: 
Proposition 6.2 If (4.5) holds, then we have

$$
\sup _{\sigma \in \mathcal{H}_{1}} \int_{M} e^{4 \pi|\sigma|^{2}} d V_{g} \leq \operatorname{Vol}(M)+\pi e^{1+4 \pi\left\langle\tau, s_{p}\right\rangle}
$$

Proof. Set $Y_{k}=\left(\tilde{U}_{k}-L_{k}\right)^{+}$, where

$$
L_{k}=\left(l_{1}, \cdots, l_{n}\right)=\sup _{\partial B_{\delta}(p)} \tilde{U}_{k}=O\left(\frac{1}{c_{k}}\right) .
$$

Then $Y_{k} \in H_{0}^{1,2}\left(B_{\delta}, \mathbb{R}^{n}\right)$, and

$$
\int_{B_{\delta}(p)}\left|\nabla_{0} Y_{k}\right|^{2} d x \leq 1-\int_{M \backslash B_{\delta}(p)}|\nabla G|^{2} d V_{g}+o_{\delta}(1) \frac{1}{c_{k}^{2}} .
$$

By Lemma 6.1 and Proposition 7.2 in Appendix, we have

$$
\int_{B_{\delta}(p)}\left|\nabla Y_{k}\right|^{2} d x \leq 1-\frac{-\frac{1}{2 \pi} \log \delta+\left\langle\tau, s_{p}\right\rangle+\epsilon(k, \delta)}{c_{k}^{2}},
$$

where $\lim _{\delta \rightarrow+0} \lim _{k \rightarrow+\infty} \epsilon(k, \delta)=0$. Let

$$
\vartheta_{k}=\frac{1}{1-\frac{-\frac{1}{2 \pi} \log \delta+\left\langle\tau, s_{p}\right\rangle+\epsilon(k, \delta)}{c_{k}^{2}}}=1+\frac{-\frac{1}{2 \pi} \log \delta+\left\langle\tau, s_{p}\right\rangle+\epsilon(k, \delta)}{c_{k}^{2}}+O\left(\frac{\log ^{2} \delta}{c_{k}^{4}}\right) .
$$

By Theorem A and the method we used to prove Corollary 3.2, we have

$$
\limsup _{k \rightarrow+\infty} \int_{B_{\delta}(p)} e^{\alpha_{k} \vartheta_{k}\left|Y_{k}\right|^{2}} d x \leq \delta^{2}(\pi e+\pi) .
$$

If $\tilde{u}_{i}<l_{k}$, we have on $B_{L r_{k}}\left(x_{k}\right)$

$$
\tau^{i}=0, \quad \tilde{u}_{i}=\frac{o_{\delta}(1)}{c_{k}}, \quad l_{k}=\frac{o_{\delta}(1)}{c_{k}} .
$$

Hence, we have $y_{k}^{i}=\tilde{u}_{k}^{i}-l_{k}+\frac{o_{\delta}(1)}{c_{k}}$ for sufficiently large $k$. A straightforward calculation shows on $B_{L r_{k}}\left(x_{k}\right)$,

$$
\vartheta_{k}\left|Y_{k}\right|^{2}=\left|\tilde{U}_{k}\right|^{2}-2 \tilde{U}_{k} L_{k}+\left|\tilde{U}_{k}\right|^{2}\left(\frac{-\frac{1}{2 \pi} \log \delta+\left\langle\tau, s_{p}\right\rangle}{c_{k}^{2}}\right)+\epsilon(\delta, k) .
$$

It is easy to check that

$$
\lim _{k \rightarrow+\infty} c_{k} L_{k}^{i}=-\frac{\left|\tau^{i}\right|}{2 \pi} \log \delta+\operatorname{sign}\left(\tau^{i}\right) s_{p}^{i}+o_{\delta}(1),
$$

and on $B_{L r_{k}}\left(x_{k}\right)$,

$$
\frac{\tilde{U}_{k}}{c_{k}}=\left(\left|\tau^{1}\right|, \cdots,\left|\tau^{n}\right|\right)+\frac{o_{k}(1)}{c_{k}}
$$

Recall that $|\tau|=1$, we get

$$
\tilde{U}_{k} L_{k}=-\frac{1}{2 \pi} \log \delta+\left\langle\tau, s_{p}\right\rangle+\epsilon^{\prime}(\delta, k)
$$


where $\lim _{\delta \rightarrow 0} \lim _{k \rightarrow+\infty} \epsilon^{\prime}(\delta, k)=0$. Since $\left|\tilde{U}_{k}\right|^{2} / c_{k}^{2} \rightarrow 1$ as $k \rightarrow+\infty$, we have

$$
\left|\tilde{U}_{k}\right|^{2} \leq \vartheta_{k}\left|Y_{k}\right|^{2}-\frac{1}{2 \pi} \log \delta+\left\langle\tau, s_{p}\right\rangle+\epsilon^{\prime}(\delta, k) .
$$

Letting $k \rightarrow+\infty$, then $L \rightarrow+\infty$, and $\delta \rightarrow 0$, we get

$$
\lim _{L \rightarrow+\infty} \lim _{k \rightarrow+\infty} \int_{B_{L r_{k}\left(x_{k}\right)}} e^{\alpha_{k}\left|\sigma_{k}\right|^{2}} d V_{g} \leq \pi e^{1+4 \pi\left\langle\tau, s_{p}\right\rangle} \lim _{L \rightarrow+\infty} \lim _{k \rightarrow+\infty} \int_{B_{L r_{k}\left(x_{k}\right)}} e^{\alpha_{k} \vartheta_{k}\left|Y_{k}\right|^{2}} d x<+\infty .
$$

Then by Corollary 5.2, we obtain

$$
\lim _{L \rightarrow+\infty} \lim _{k \rightarrow+\infty} \int_{B_{\rho}(p) \backslash B_{L r_{k}}\left(x_{k}\right)} e^{\alpha_{k}\left|\sigma_{k}\right|^{2}} d V_{g}=\left|B_{\rho}\right|, \quad \forall \rho>0 .
$$

Clearly,

$$
\left|Y_{k}\right|^{2} \leq\left|\tilde{U}_{k}\right|^{2}=\left|\sigma_{k}\right|^{2}, \quad \text { and } \quad \alpha_{k} \vartheta_{k}\left|Y_{k}\right|^{2} \leq \alpha_{k}\left|U_{k}\right|^{2}-2 \log \delta+C \text {. }
$$

Hence

$$
\lim _{L \rightarrow+\infty} \lim _{k \rightarrow+\infty} \int_{B_{\rho}(p) \backslash B_{L r_{k}}\left(x_{k}\right)} e^{\alpha_{k} \vartheta_{k}\left|Y_{k}\right|^{2}} d x \leq O\left(\delta^{-2}\right) \int_{B_{\rho}(p)} e^{\alpha_{k}\left|\sigma_{k}\right|^{2}} d V_{g}=O\left(\delta^{-2}\right)\left|B_{\rho}(p)\right| .
$$

It is easy to see that for any fixed $\rho>0$

$$
\int_{B_{\delta}(p) \backslash B_{\rho}(p)} e^{\alpha_{k} \vartheta_{k}\left|Y_{k}\right|^{2}} d V_{g} \rightarrow\left|B_{\delta} \backslash B_{\rho}\right| .
$$

Letting $\rho \rightarrow 0$, we get

$$
\lim _{L \rightarrow+\infty} \lim _{k \rightarrow+\infty} \int_{B_{\delta}(p) \backslash B_{L r_{k}}\left(x_{k}\right)} e^{\alpha_{k} \vartheta_{k}\left|Y_{k}\right|^{2}} d x=\pi \delta^{2}, \lim _{L \rightarrow+\infty} \lim _{k \rightarrow+\infty} \int_{B_{L r_{k}}\left(x_{k}\right)} e^{\alpha_{k} \vartheta_{k}\left|Y_{k}\right|^{2}} d x \leq \delta^{2} \pi e .
$$

Then the Proposition follows from (6.1) and Corollary 5.2.

\section{Appendix}

We will prove two propositions which have been used in section 5 and section 6 . Since the proof is routine, we prove them in this appendix. The first proposition is an extension of Theorem 2.2 in [S2]:

Proposition 7.1. Let $\sigma$ be a smooth section of $E$ with the equation

$$
\Delta \sigma=f .
$$

If $\|\sigma\|_{L^{2}} \leq \gamma$ and $\|f\|_{L^{1}} \leq 1$, then for any $q<2$, there is a constant $C(q)$ which depend only on $q \gamma$ and $M$, s.t.

$$
\|\sigma\|_{H^{1, q}} \leq C(q)
$$

Proof. For any $p \in M$, we take a cut-off function which is 1 in $B_{r}$ and 0 outside $B_{2 r}$. Write $\sigma=u^{i} e_{i}$. Given $t>0$, we set $u^{i, t}=\min \left\{\eta u^{i}, t\right\}$ and $\sigma^{t}=u^{i, t} e_{i}$. Then

$$
\int_{M}\left\langle f, \eta^{2} \sigma^{t}\right\rangle d V_{g}=\int_{M}\left\langle\eta^{2} \sigma^{t}, \Delta \sigma\right\rangle d V_{g} .
$$

We get

$$
\int_{B_{r}(p)} \sum_{i}\left|\nabla \eta u^{i, t}\right|^{2} d x \leq C_{1} t+C_{2} \leq C^{\prime} t
$$

when $t$ is sufficiently large. 
Let $u_{*}^{i}$ be the rearrangement of $\eta u^{i}$, and $\mu_{\mathbb{R}^{2}}\left(B_{\rho}\right)=\mu_{\mathbb{R}^{2}}\left\{\eta u^{i, t} \geq t\right\}$. Then we have

$$
\inf \left\{\int_{B_{r}}|\nabla v|^{2} d x: v \in H_{0}^{1,2}\left(B_{r}\right) \text { and }\left.v\right|_{B_{\rho}}=t\right\} \leq C t .
$$

On the other hand, the inf is achieved by $-t \log \frac{|x|}{r} / \log \frac{r}{\rho}$. By a direct computation, we have

$$
\begin{gathered}
\frac{2 \pi t}{\log \frac{r}{\rho}} \leq C, \\
\mu_{M}\left(\left\{x \in B_{r}: \eta u^{i} \geq t\right\}\right) \leq C \mu_{\mathbb{R}^{n}}\left(\left\{x \in B_{r}: \eta u^{i} \geq t\right\}\right)=C \mu_{\mathbb{R}^{n}}\left(B_{\rho}\right) \leq C(r, p) e^{-A(r, p) t} .
\end{gathered}
$$

Hence, we can find a constant $A$, s.t.

$$
\left|\left\{\eta u^{i} \geq t\right\} \cap B_{r}\right| \leq e^{-A t} .
$$

In the same way, we get

$$
\left|\left\{\eta u^{i} \leq-t\right\} \cap B_{r}\right| \leq e^{-A^{\prime} t} .
$$

By the compactness of $M$, we get

$$
|\{|\sigma| \geq t\}| \leq e^{-\delta_{0} t}
$$

for some $\delta_{0}$ and sufficiently large $t$. Then, for any $\delta<\delta_{0}$,

$$
\int_{M} e^{\delta|\sigma|} d V_{g} \leq \sum_{m=0}^{\infty} \mu(\{m \leq u \leq m+1\}) e^{\delta(m+1)} \leq \sum_{m=0}^{\infty} e^{-\left(\delta_{0}-\delta\right) m} e^{\delta} \leq C .
$$

Locally, we denote $U=\left(u^{1}, \cdots, u^{n}\right)$, then

$$
\Delta_{0} u^{i}=f^{i}+T_{1}^{i}(\nabla U)+T_{2}^{i}(U),
$$

where $T_{1}$ and $T_{2}$ are smooth and linear at each point. Testing the above equation with the function $\eta^{2} \log \frac{1+2 u^{i+}}{1+u^{i+}}$, where $\eta$ is 1 on $B_{r}$ and 0 outside $B_{2 r}$. We obtain the following

$$
\int_{B_{r}} \sum_{i=1}^{n} \frac{\left|\nabla_{0} \eta u^{i+}\right|^{2}}{\left(1+2 u^{i^{+}}\right)\left(1+u^{i^{+}}\right)} d x \leq \log 2+\int_{B_{r}}\left(T_{1^{\prime}}^{i}\left(\nabla \eta U^{+}\right)+T_{2^{\prime}}^{i}\left(U^{+}\right)\right) d V_{g},
$$

where $T_{1^{\prime}}$ and $T_{2^{\prime}}$ are smooth and linear at each point. Since $\int_{M} e^{\delta|\sigma|} d V_{g}<C(\delta)$, we have

$$
\int_{B_{r}} \sum_{i=1}^{n} \frac{\left|\nabla u^{i^{+}}\right|^{2}}{\left(1+2 u^{i^{+}}\right)\left(1+u^{i+}\right)} \leq C
$$

Given $q<2$, by Young's inequality, we have

$$
\begin{aligned}
\int_{B_{r}}\left|\nabla u^{i+}\right|^{q} d V_{g} & \leq \int_{B_{r}}\left(\frac{\left|\nabla_{0} u^{i+}\right|^{2}}{\left(1+u^{i+}\right)\left(1+2 u^{i+}\right)}+\left(\left(1+u^{i+}\right)\left(1+2 u^{i+}\right)\right)^{\frac{q}{2-q}}\right) d x \\
& \leq \int_{B_{r}}\left(\frac{\left|\nabla_{0} u^{i+}\right|^{2}}{\left(1+u^{i+}\right)\left(1+2 u^{i+}\right)}+C e^{\delta u^{i+}}\right) d x .
\end{aligned}
$$

In the same way,

$$
\int_{B_{r}}\left|\nabla u^{i^{-}}\right|^{q} d V_{g}<C(q)
$$

Again, by the compactness of $M$, we get

$$
\|\sigma\|_{H^{1, q}}<C(q)
$$

for some $C(q)$. 
On $\Omega_{p}$, we can set

we have the following Lemma:

$$
G=-\frac{\log r}{2 \pi} \tau+s_{p}+\theta
$$

Lemma 7.2. There are constants $\gamma \in(0,1)$ and $A>0$, s.t.

$$
|\nabla \theta|(x) \leq A r^{\gamma-1}
$$

when $x$ is near 0 . Therefore, we have

$$
\int_{M \backslash B_{\delta}(p)}|\nabla G|^{2} d V_{g}=-\frac{1}{2 \pi} \log \delta+\left\langle\tau, s_{p}\right\rangle+o_{\delta}(1) .
$$

Proof. Let $\theta=v^{i} e_{i}$, and $V=\left(v^{1}, \cdots, v^{n}\right)$, we have the equation of $V$

$$
-\Delta_{0} V=-\nabla Q_{1} \nabla V+\frac{1}{r} Q_{2} \frac{\partial Q_{3}}{\partial r}+F,
$$

where $Q_{1} Q_{2}$ and $Q_{3}$ are smooth matrix, $F$ is a smooth vector function . Hence $V \in W_{l o c}^{2, p}$ for any $p<2$.

Clearly, we can write

$$
Q^{-1} \frac{\partial Q}{\partial r}=Q^{-1} \frac{\partial Q}{\partial x_{i}} \frac{x_{i}}{r}=\Lambda_{i} \frac{x_{i}}{r}+F_{1},
$$

where $\Lambda_{k}$ are constant vector, and $F_{1}=o(1)$. Hence

$$
-\Delta_{0} V=\Lambda_{i} \frac{x_{i}}{r^{2}}+F_{2}
$$

where $F_{2} \in L_{l o c}^{\infty}$. Then

$$
-\Delta_{0} x_{m} V=\Lambda_{i} \frac{x_{i} x_{m}}{r^{2}}+x_{m} F_{2}+\frac{\partial V}{\partial x_{m}} \in L_{l o c}^{q}
$$

for any $q>0$. Hence, we get

$$
\left\|x_{m} V\right\|_{C^{1}\left(B_{r}\right)} \leq\|V\|_{C^{0}\left(B_{2 r}\right)} 2 r+C\left(1+\|\nabla V\|_{\left.L^{2 q}\right)} r^{\frac{1}{q^{\prime}}}\right.
$$

for any $q>1$. Notice that $V(0)=0$, and then $V=O\left(r^{\gamma}\right)$ for some $\gamma>0$, we get

$$
\left|x_{m} \frac{\partial V}{\partial x_{i}}(x)\right| \leq C r^{\gamma}
$$

for some $\gamma>0$, any $i, m=1,2$ and $x \neq 0$. In the end, we get

$$
|\nabla V|(x) \leq|x|^{\gamma-1}
$$

\section{REFERENCES}

[A] T. Aubin: Some Nonlinear Problems in Riemann Geometry. Spinger, 1998.

[C-C] L. Carleson and A. Chang: on the existence of an extremal function for an inequality of J.Moser. Bull. Sc. Math., 110 (1986) 113-127.

[C] A. Chang: The Moser-Trudinger inequality and applications to some problems in conformal geometry. Nonlinear partial differential equations in differential geometry (Park City, UT, 1992), 65-125, IAS/Park City Math. Ser., 2, Amer. Math. Soc., Providence, RI, 1996.

[C-Y] A. Chang and P. Yang: Conformal deformation of metrics on $S^{2}$. J. Differential Geometry, 27 (1988) 259-296. 
[C-L] W. Chen and C. Li: Classification of solutions of some nonlinear elliptic equations. Duke Math. J., 63 (1991) 615-622.

[Ch] Cherrien: Une inégalité de Sobolev sur les variétés Riemanniennes. Bull. Sc. Math, 103 (1979) 353-374.

[D-J-L-W] W. Ding, J. Jost, J. Li and G. Wang: The differential equation $-\Delta u=8 \pi-8 \pi h e^{u}$ on a compact Riemann Surface. Asian J. Math., 1 (1997) 230-248.

[D-J-L-W2] W. Ding, J. Jost, J. Li and G. Wang: Multiplicity results for the two-vortex Chern-Simons Higgs model on the two sphere. Commun. Math. Helv., 74 (1999) 118-142.

[D-J-L-W3] W. Ding, J. Jost, J. Li and G. Wang: Existence results for mean field equations. Ann. Inst. Henri Poincaré, Analyse non linéaire, 16 (1999) 653-666.

[F] L. Fontana: Sharp borderline Sobolev inequalities on compact Riemannian manifolds. Comm. Math. Helv., 68 (1993) 415- 454.

[J-W] Jackiw, R. and Weinberg, E., Self-dual Chern-Simons vortices. Phys. Rev. Lett., 64 (1990) $2234-2237$.

[L] Y. Li: Moser-Trudinger inequality on compact Riemannian manifolds of dimension two. J. Partial Differential Equations 14 (2001) 163-192.

[L-L] Y. Li and P. Liu: A Moser-Trudinger inequality on the boundary of a compact Riemann surface. Math. Z., 250 (2005) 363-386.

[L-Y] Y. Li and Yang. Li: Extremal function for Adams-Fontana inequality in 4-manifold. In prepairation.

[M] J. Moser: A sharp form of an Inequality by N.Trudinger. Ind. Univ. Math. J., 20 (1971) 1077-1091.

[S] M. Struwe: Critical points of embedding of $H_{0}^{1, n}$ into Orlicz space. Ann. Inst. Henri. Poincare 5 (1988) 425-464.

[S2] M. Struwe: Positive solution of critical semilinear elliptic equations on non-contractible planar domain. J. Eur. Math. Soc., 2 (2000) 329-388.

[T] N. S. Trudinger: On embedding into Orlicz space and some applications. J. Math. Mech. 17 (1967) $473-484$.

[Y] Y. Yang: A weighted form of Moser-Trudinger inequality on Riemannian surface. Nonlinear Analysis (2005), in press.

Yuxiang Li

ICTP, Mathematics Section, Strada Costiera 11, I-34014 Trieste, Italy

E-mail address: liy@ictp.it

Pan Liu

Department of Mathematics, East China Normal University, 3663, Zhong Shan North Rd, Shanghai 200062, P.R.China

E-mail address: pliu@math.ecnu.edu.cn

\section{Yunyan Yang}

Department of Mathematics, Information School, Renmin University of China, Beijing 100872, P. R. China

E-mail address: yunyan_yang2002@yahoo.com.cn 